Optimization of the cooling profile to achieve crack-free $\mathrm{Yb}$ :S-FAP crystals

H.S. Fang, S.R. Qiu, L.L. Kheng, K.I. Schaffers, J.B. Tassano, J.A. Caird, H. Zhang

August 24, 2007

Journal of Crystal Growth 
This document was prepared as an account of work sponsored by an agency of the United States Government. Neither the United States Government nor the University of California nor any of their employees, makes any warranty, express or implied, or assumes any legal liability or responsibility for the accuracy, completeness, or usefulness of any information, apparatus, product, or process disclosed, or represents that its use would not infringe privately owned rights. Reference herein to any specific commercial product, process, or service by trade name, trademark, manufacturer, or otherwise, does not necessarily constitute or imply its endorsement, recommendation, or favoring by the United States Government or the University of California. The views and opinions of authors expressed herein do not necessarily state or reflect those of the United States Government or the University of California, and shall not be used for advertising or product endorsement purposes. 


\title{
Optimization of the cooling profile to achieve crack-free Yb:S-FAP crystals
}

\author{
H.S. Fang ${ }^{1}$, S. R. Qiu ${ }^{2}$, L.L. Zheng ${ }^{1}$, K.I. Schaffers ${ }^{2}$, J.B. Tassano ${ }^{2}$, J.A. Caird, H. Zhang ${ }^{* 1}$ \\ ${ }^{1}$ Department of Mechanical Engineering State University of New York at Stony Brook \\ ${ }^{2}$ Lawrence Livermore National Laboratory, 7000 East Ave., Livermore, CA 94551
}

\begin{abstract}
$\mathrm{Yb}: \mathrm{S}-\mathrm{FAP}\left[\mathrm{Yb}^{3+}: \mathrm{Sr}_{5}\left(\mathrm{PO}_{4}\right)_{3} \mathrm{~F}\right]$ crystals are an important gain medium for diode-pumped laser applications. Growth of $7.0 \mathrm{~cm}$ diameter Yb:S-FAP crystals utilizing the Czochralski (CZ) method from $\mathrm{SrF}_{2}$-rich melts often encounter cracks during the post growth cool down stage. To suppress cracking during cool down, a numerical simulation of the growth system was used to understand the correlation between the furnace power during cool down and the radial temperature differences within the crystal. The critical radial temperature difference, above which the crystal cracks, has been determined by benchmarking the simulation results against experimental observations. Based on this comparison, an optimal three-stage ramp-down profile was implemented and produced high quality, crack-free Yb:S-FAP crystals.
\end{abstract}

PACS: 61.72.-y; 71.55.-i; 81.10.Fq; 81.10.Aj

Key words: Laser crystal, cracking, thermal stress, optimization, numerical simulation

*Corresponding author. Tel. 631-632-8492. Fax: 631-632-8544. Email: Hui.Zhang@sunysb.edu 


\section{Introduction}

Yb-S-FAP crystals have many attractive laser properties making them well suited for diode pumping in moderate thermal load applications [1]. Currently, $7.0 \mathrm{~cm}$ diameter $\mathrm{Yb}$ : S-FAP crystals are being grown by using the Czochralski method from a $\mathrm{SrF}_{2}$-rich melt $[2,3]$. The growth of these crystals offers several challenges, which were resolved for $3.5 \mathrm{~cm}$ diameter. These included cracking, cloudiness [4-6], melt inclusions [5], anomalous absorption [7], and low-angle grain boundaries [8]. Specifically relating to this research, cracking was eliminated by reducing defects, cooling crystals using mono-linear cool-down power profile and leaving the crystal in contact with the melt during cooling. However, in scaling to $7.0 \mathrm{~cm}$ diameter, modifications to this procedure are required to prevent cracking.

Crystal cracking during cool down is directly related to the buildup of thermal stress and associated strains. Since Yb:S-FAP crystals have cylindrical symmetry, for simplicity of modeling, the total thermal stress within the crystal boule can be characterized by the radial and axial components. There are critical thermal stresses along both directions, above which, the crystal will be subject to cracking and it is believed that these critical values are temperature dependent. For example, at the beginning of the cool down when the temperature of the furnace is high, the crystal can usually sustain a large temperature difference in the radial direction without cracking. It should be noted that defects such as bubble core may significantly affect the critical values and cause them to be lower than modeled. Thus, depending on the level and distribution of thermal stress during the cool down, cracks can happen within the crystal along axial, radial or both directions.

In situ measurements of the temperature distribution within the crystal and its 
surroundings during growth and cool-down are not practical in this case and significantly disturb the system thermodynamics. Therefore, numerical simulations of the growth and post-growth cool down are an ideal alternative to understanding the fundamental causes for issues related to crystal quality. In addition, numerical simulation of the growth furnace makes it possible to predict the temperature distribution within the crystal and its surroundings, temperature gradients within the furnace, and the relationship of these components with the power applied during the growth and cool down processes. Simulation results can be benchmarked against experimental observations to optimize growth and cool down parameters for growing high quality crystals with suppressed cracking.

Brice [9] examined thermal strain in the crystal during growth and cooling, and obtained the analytical solution for the maximum allowed cooling rate. Related to the maximum cooling rate, there exists a critical radial temperature difference between the center and the edge of the crystal above which the crystal will crack. This analysis was acceptable for crystals with a small value of ratio of diameter to length and weak radiation heat losses [10]. Recently, Metzger and Backofen [11] simulated the annealing process for GaAs crystal growth. They showed that the evolution of the maximum stress was strongly correlated to the radial temperature difference between the center and the edge of the crystal. The predicted radial temperature differences across the crystal by one- and two-dimensional models displayed similar behavior. Furthermore, a one-dimensional model was employed to optimize the cooling process in which the radial temperature difference was required to be limited by a critical value. The above analysis established the relationship between radial temperature difference and thermal strain induced in the cylindrical crystal. Although this paper 
concentrated on modeling the growth of GaAs, not an optical material, a similar approach may be applicable to the current study for Yb:S-FAP crystals.

\section{Methodology}

A numerical model has been developed to simulate the temperature profile of the $\mathrm{Yb}$ :S-FAP crystal grown in the CZ furnace shown in Figure 1. The radio-frequency (RF) induction heating method is used [12-15] for a standard oxide-type furnace. Figure 2 shows the configuration of the crucible and crystal position during cool down. In this Figure, $R$ is the radius of the crystal, $T(0)$ and $T(R)$ are temperatures at the center and surface of the crystal, respectively, $T_{c}$ is the crucible temperature, and $T_{\text {sur }}$ is the crystal surrounding temperature that is measured at the center between the crystal surface and the inner diameter of the Ir ring as shown.

Simulations were performed first to examine the effect of internal radiation from the crystal during cool down to determine the conditions under which the crystal cracks. The numerical model is then used to optimize the cooling profile by ensuring that the thermal stresses in the crystal are maintained within a critical value throughout cooling. While both radial and axial temperature gradients may contribute to cracking, for simplicity, the current study considers that cracks only originate from the radial temperature gradient. Simulation results from the one dimensional model suggest that the crystal can be cooled faster at high temperature but must be cooled at a slower rate at lower temperatures.

\subsection{Relationship between power level and crystal temperature}

To determine the optimal cool-down profile, the proper relationship between the heating power and temperature distribution within the crystal growth furnace needed to be derived. 
A simplified cooling model has been built based on dynamic models and process control discussed in references 11, and 16-19. Although this analysis method does not consider all parameters, it effectively reflects the major characteristics of the growth system. Process control for the experimental growth system is treated as a simple dynamic system with the system energy as the input and the average system temperature $T_{f}$, as the output. The system energy is provided by RF induction heating and the heat loss is restricted to the environment (with temperature $T_{\infty}$ ) only. Thus the following equation can be used to describe the energy balance of the system,

$$
m c_{p} \frac{d T_{f}}{d t}=P-h_{e f f, f} A_{f}\left(T_{f}-T_{\infty}\right)
$$

where $P$ is the power of the RF induction heater, $m$ is the mass of the furnace, $c_{p}$ is the heat capacity of the furnace, $h_{\text {eff } f}$ is the effective heat transfer coefficient between the furnace and environment around the furnace, and $A_{f}$ is the total surface area of furnace. Because $T_{f}$ is hard to define, it is replaced by the gas temperature surrounding the crystal, $T_{\text {sur }}$, by approximation without missing major characteristics. Therefore, Equation (1) can be rewritten as

$$
\left(m c_{p}\right)^{\prime} \frac{d T_{\text {sur }}}{d t}=P-\left(h_{e f f, f} A_{f}\right)^{\prime}\left(T_{s u r}-T_{\infty}\right)
$$

where $\left(m c_{p}\right)^{\prime}$ and $\left(h_{e f f, f} A_{f}\right)^{\prime}$ having different values from that of $m c_{p}$ and $h_{e f f, f} A_{f}$. For a steady state, $\mathrm{dT}_{\text {surf }} / \mathrm{dt}$, is zero, and $\left(h_{\text {eff }, f} A_{f}\right)^{\prime}$ can be obtained from $\left(h_{e f f, f} A_{f}\right)^{\prime}=\frac{P}{T_{s u r}-T_{\infty}}$ for any given power. Furthermore, by monitoring $T_{\text {sur }}$ as a function of time and power, the value of $\left(m c_{p}\right)^{\prime}$ can also be determined by equation (2). In this paper, $\left(m c_{p}\right)^{\prime}$ and 
$\left(h_{e f f, f} A_{f}\right)^{\prime}$ are assumed to be constant for simplification. They can be improved by describing them as a time-dependent function for more detailed analysis.

As the cooling is initiated, the power level is turned down slowly. The power ramp-down rate is represented as $\Delta P / \Delta t_{0}$, where $\Delta t_{0}$ is a constant for experiments. In the model optimization, $\Delta P$ must be determined at every step so as to control the induced temperature difference. Since the effective thermal conductivity, $k_{\text {eff }}$, is a strong function of temperature, and the crystal surrounding temperature, $T_{\text {sur }}$, is a function of the power level, no analysis solution is therefore possible. Within each time step, $\Delta t_{0}$, the power level, $P$, can be treated as a constant; Equation (2) can be rewritten as follows

$$
\frac{d \theta}{d t}=-\chi \theta, \quad \text { at each time step }
$$

where $\theta=P /\left(h_{e f f, f} A_{f}\right)^{\prime}-\left(T_{\text {sur }}-T_{\infty}\right)$ and $\chi=\left(h_{\text {eff }, f} A_{f}\right)^{\prime} /\left(m c_{p}\right)^{\prime}$. From Equation (3) with initial condition, the relationship between $P$ and $T_{\text {sur }}$ can be established. At the end, the following relationship can be obtained,

$$
\Delta P=\overline{h_{\text {eff }} A_{f}}\left[\Delta T_{\text {sur }}+\int_{t}^{t+\Delta t_{o}} \lambda(t) d t\right], \quad \text { for each time step }
$$

where $\lambda(t)=\theta_{0} \chi e^{-\chi t}$. It is apparent from the equation that as heating power is ramped down, the system temperature is reduced, so does the.surrounding temperature. To make the optimization procedure simpler, we further approximate the relationship between $\Delta P$ and $\Delta T_{\text {sur }}$ as $\Delta P=\overline{h_{e f f} A_{f}} \Delta T_{\text {sur }}$,

where $\overline{h_{\text {eff }, f} A_{f}}$ can be obtained from the experimental data. Equation (3) can also be used in the optimization. However, more data such as $\overline{m c_{p}}$ is required. The effective convection

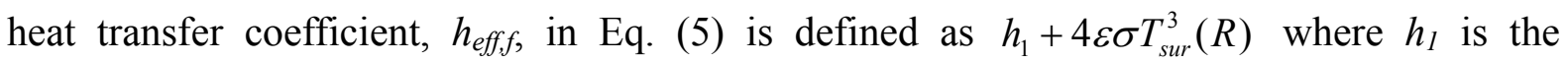


convection heat transfer coefficient. Since the growth system is complex, many simplifications have been made to get Eq. (5). Further improvement of the analysis can be made through data gained by both experiment and simulation.

\subsection{Cooling of crystal from surface}

For CZ crystal growth, several radiation models are available[20] to relate crystal temperature to ramp-down power. For simplification, the effective convection heat transfer coefficient is used to take into account of convection, radiation effects. Thus the energy balance equation on the crystal surface can be written as

$$
\begin{aligned}
P_{l}(t) & \approx h_{2}\left[T(R, t)-T_{\text {sur }}(t)\right]+\varepsilon F_{c f} \sigma\left[T^{4}(R, t)-T_{\text {sur }}^{4}(t)\right] \\
& \approx\left[h_{2}+4 \varepsilon F_{c f} \sigma T^{3}(R)\right]\left[T(R, t)-T_{\text {sur }}(t)\right]=h_{2, e f f}\left[T(R, t)-T_{\text {sur }}(t)\right]
\end{aligned}
$$

where $P_{l}$ is the heat loss from the crystal surface to the surrounding gas, $F_{\mathrm{cf}}$ is the view factor from the crystal to its surrounding, $h_{2}$ is the convection heat transfer coefficient, and $\varepsilon$ is the emissivity of the crystal surface. Equation (6) will be used to determine the boundary conditions for the crystal for crystal temperature calculations.

\subsection{Calculation of crystal radial temperature difference}

Experimental results show that crystal cracking normally occurs at the Iridium ring where the temperature gradients in the crystal are the largest. Thus we assume this area along the length of the crystal dominates the cooling process and the radial temperature difference can be chosen as the control parameter. During cooling, the power level is reduced in time duration, $\Delta t_{0}$. In the current study, $\Delta t_{0}$ is chosen as five minutes, indicating the induction heating power is adjusted every five minutes. A one-dimensional energy equation together with boundary and initial conditions can be written as, 
$\frac{1}{r}\left[\frac{\partial}{\partial r}\left(r k \frac{\partial T}{\partial r}\right)\right]=\frac{\partial\left(\rho c_{p} T\right)}{\partial t}, \quad 0 \leq r \leq R, \quad t_{0}<t \leq t_{0}+\Delta t_{0}$,

$\frac{\partial T}{\partial r}+\frac{h_{2, e f f}}{k_{\text {eff }}}\left(T-T_{\text {sur }}\right)=0, \quad r=R, \quad t_{0}<t \leq t_{0}+\Delta t$,

$T=T\left(t_{0}, r\right), \quad 0 \leq r \leq R, \quad t=t_{0}$,

where $h_{2, \text { eff }}$ is the effective convection heat transfer coefficient of the gas surrounding the crystal and $k_{\text {eff }}$ is the effective thermal conductivity of the crystal. In the boundary conditions for Eq.(8) the crystal surface heat loss from Eq.(6) is applied. Since Yb:S-FAP is a transparent optical material, inner radiation is high, particularly when the crystal temperature is high, so the internal radiation can be approximated by the simplest Rosseland model [21] using the effective thermal conductivity.

$$
k_{\text {eff }}=k_{s}+k_{r}, k_{r}=\frac{16 n^{2} \sigma T^{3}}{3 a_{R}},
$$

where $k_{s}$ is the thermal conductivity of the crystal, $k_{r}$ is the portion of thermal conductivity from radiation, $n$ is the refractive index of the surrounding gas, $\sigma$ is the Stefan-Boltzmann constant, and $\alpha_{R}$ is the Rosseland mean absorption coefficient. The value of effective thermal conductivity depends strongly on the crystal temperature as shown in Fig. 3. In our simulation, $\alpha_{R}$ is set to 100 .

Assume thermal properties may not change within every power control interval, e.g., five minutes, the analytical solution of Eq. (9) is obtained as [22]

$$
T(r, t)=\frac{2}{R^{2}} \sum_{m=1}^{\infty} e^{-\alpha \beta_{m}^{2} t} \frac{\beta_{m}^{2} J_{0}\left(\beta_{m} r\right)}{\left[\beta_{m}^{2}+\left(h_{2, e f f} / k_{e f f}\right)^{2}\right] J_{0}^{2}\left(\beta_{m} R\right)} \int_{0}^{R} r^{\prime} J_{0}\left(\beta_{m} r^{\prime}\right) T\left(t_{0}, r^{\prime}\right) d r^{\prime}
$$

where $\alpha$ is the thermal diffusivity, $J_{0}(x)$ is the Bessel function, and $\beta_{m}$ represents the positive roots of 
$\beta_{m} J_{0}^{\prime}\left(\beta_{m} R\right)+\left(h_{2, e f f} / k_{\text {eff }}\right) J_{0}\left(\beta_{m} R\right)=0$

Since Eq.(12) is an infinite series, it is necessary to keep the first several terms as an approximation, which is accurate when the Biot number is not large. Based on the analytical solution of the temperature distribution, the relationship between the radial temperature difference and power ramp down rate can be established.

\subsection{Critical radial temperature}

In order to determine the optimal cooling profile that prevents the crystal from cracking during cool down, the simulation model must be validated. First, the value of the critical radial temperature above which cracking will occur is required. Since this value is not readily available for $\mathrm{Yb}$ :S-FAP crystals, it is estimated through simulations and verified by experiments bearing mono-linear and bi-linear cooling profiles, respectively. The assumed critical radial temperature difference in the simulation is shown in Figure 4. The characteristics of the assumed values are based on the belief that the critical values are temperature dependent and are small at lower temperatures and large at higher temperatures.

An initial power change is assumed to perform the optimization. The crystal temperature distribution is then simulated by applying Eqs. (1)-(12), where the crystal radial temperature differences are calculated and compared with the critical values. If the predicted crystal radial temperature difference is larger than that of the critical differences, the power change used in the current time step has to be reduced in order to limit the thermal stress in the radial direction within the crack free regime. This procedure is reiterated until a proper power ramp down profile is obtained such that the achieved radial temperature difference equals the critical value. The optimizing procedures are summarized in Figure 5. It is noted that the 
radial temperature difference in crystal should be maintained at a level smaller than the critical radial temperature difference to avoid cracking. The mathematical formulae for this constraint is described as follows,

$\Delta T_{R}=T(0)-T(R) \leq \Delta T_{R, c r i}$

\section{Results and Discussion}

The most difficult part of the modeling is to define material properties. Table 1 lists the material properties that were used in this simulation [3, 23]. The assumed critical radial temperature difference is shown in Figure 4. The furnace surface area $A_{f}$ is approximated to be $0.67 \mathrm{~m}^{2}$. The convection heat transfer coefficient $h_{1}$ is set to a small value of $4 \mathrm{~W} / \mathrm{m}^{2} \mathrm{~K}$. The effective heat transfer coefficient in Eq. (5) is given as $20 \mathrm{~W} / \mathrm{m}^{2} \mathrm{~K}$. The initial crystal temperature is chosen to be $1800 \mathrm{~K}$, lower than the $2059 \mathrm{~K}$ melt temperature, based on the fact that the average crystal temperature is lower than the melt temperature at the start of the cool down.

Actual temperature and temperature differences along the radial directions were simulated for a system that was cooled under a mono-linear forty-eight-hour cooling profile. The power level during the cooling period and its corresponding crystal surrounding temperatures are shown in Figure 6 (a). The dashed-dotted line in Figure 6 (b) shows the assumed critical radial temperature difference. The solid line represents the radial temperature differences during cool down. It is seen that the temperature difference along the radial direction increases with time. The solid curve intercepts the critical value at 37 hours 
suggesting that, under the current assumption, after 37 hours, the crystal is susceptible to cracking. Note that the radial temperature could reach $50 \mathrm{~K}$ when the power level is decreased to $0 \%$. The predicted crack zone is marked by the hatched solid lines.

Similar calculations were done with a system that is cooled under a ninety-six hour bi-linear cooling profile. The calculated results are plotted in Figures 7(a) and (b). In Figure 7(b), the crystal is predicted to crack after 78 hours of cooling. Note that the radial temperature could reach $36 \mathrm{~K}$ when the power level is decreased to $0 \%$. The crack zone is marked with the green solid lines.

Under both cooling profiles, the temperature difference between the center and the edge of the crystal is small at the beginning, but reaches very high values at lower temperatures. This phenomenon can be attributed to the fact that the effective thermal conductivity within the crystal is significantly reduced at lower temperatures (Figure 3). Therefore, while the surface temperature is reduced due to the heat loss from convection and radiation, the temperature at the center can still be very high suggesting that it is necessary to reduce the cooling rate at low temperatures to allow for better temperature equilibration between the center and outside of the crystal to suppress cracking.

The observed time at which the crystal starts to crack in the experiments agrees well with the predicted values. The crystals harvested under the two cooling profiles discussed above are shown in Figures 8(a) and 8(b). These agreements between the model and experiment ensure that the assumed values and characteristics of the critical radial temperature difference are valid. It further verifies that the analytical approaches, and other selected parameters in the model are reasonable. Moreover, the apparent improvement of 
crystal cracking under the bi-linear cooling profile suggests that a multi-linear cooling profile is ideal.

The optimized multi-linear power ramp-down profile is shown in Figure 9 (a) along with the crystal surrounding temperature. It is apparent that the power drops at a much faster rate when the surrounding temperature is high and slows down as the surrounding temperature decreases. Such behavior is caused by the significant reduction in the effective thermal conductivity with decreasing temperature (see Figure 3). The calculated radial temperature difference in the crystal during cooling is shown in Figure 9 (b) and due to the optimized power ramp-down profile, the values are nearly identical to that of the critical temperature difference. Importantly, from the data in Table 2, it is evident that as the temperature of the crystal decreases, the radial temperature difference also decreases due to the slower cooling rate at the lower temperatures.

For the purpose of practical furnace programming, a tri-linear power ramp-down profile is preferred over the calculated profile for cooling the crystal. Utilizing the same procedures that produced Figure 9, an optimized tri-linear ramp-down profile is obtained as shown in Figure 10 (a). The crystal surrounding temperature and radial temperature difference are also shown in Figure 10. To satisfy the critical temperature difference constraint, an extended cool down time of 150 hours is required in comparison to that of around 100 hours for the continuous ramp-down profile. At 150 hours, the power level is $2 \%$ and the temperature difference is about 1 degree, however, the crystal temperature is still relatively high at $720 \mathrm{~K}$. If the power is shut down at this time the crystal cooling rate will be high and a spike will occur in the temperature difference as shown in Figure 10 (b). It 
has been experimentally observed that the crystals will occasionally crack if the power is turned off at the 150 hour cool down point, making it necessary to wait for a period of time before shutting off the power at the end of the ramp down so that the temperature difference can reach its minimum. In the growth experiment, the power control system can only be adjusted down to within $2.5 \%$ of the full power level and the power supply is then turned off. This parameter has also been implemented in the simulation resulting in a spike for the radial temperature difference at power of $2.5 \%$ (Figure 10 (b)). The crystal surrounding temperature is still rather high at $2.5 \%$ in comparison to room temperature, Figure 10 (a), when the power is turned off and the surrounding temperature will change to room temperature rapidly through normal thermal dissipation. When it exceeds the critical temperature gradient value, cracks may develop at the end of the cool down, which have been observed.

In conclusion, a tri-linear power ramp-down profile has been utilized to routinely harvest $7.0 \mathrm{~cm}$ diameter $\mathrm{Yb}: \mathrm{S}-\mathrm{FAP}$ crystals without cracking in the upper useful portion of the crystal (Figure $8(\mathrm{c})$ ). The cracking has been significantly reduced in comparison to the boules with alternate cool down profiles as shown in Figures $8(a-b)$. Cracks seen at the bottom of the crystal result from a strong thermal perturbation caused by solidification and cracking initiated within the solidified melt. Further study is underway to eliminate this cracking as well.

\section{Summary}

A numerical model has been developed to predict the temperature distributions in the $\mathrm{CZ}$ system used to grow Yb:S-FAP crystals. Simulations have been benchmarked against 
experiment for both mono-linear and bi-linear ramp-down profiles. These results suggest that cracking occurs when radial temperature differences within the crystal exceed that of the critical value. Based on this result, an optimized three-stage power ramp-down profile was proposed and resulted in high-quality crack-free, $7.0 \mathrm{~cm}$ diameter Yb:S-FAP crystals. This success demonstrates the advantages of combining numerical simulation with experiment to improve crystal quality in $\mathrm{CZ}$ crystal growth systems and reduce process development time.

\section{Acknowledgements}

This work was performed under the auspices of U.S. DOE by the University of California, Lawrence Livermore National Laboratory under contract No. W-7405-Eng-48. 


\section{References}

1. $\quad$ Schaffers, K.I., Yb:S-FAP lasers. Optical Materials, 2004. 26(4): p. 391-394.

2. Schaffers, K.I., et al., Progress in the growth of $Y b$ : S-FAP laser crystals. Journal of Crystal Growth, 2001. 225(2-4): p. 449-453.

3. Schaffers, K.I., et al., Growth of Yb: S-FAP [Yb3+:Sr5(PO4)3F] crystals for the Mercury laser. Journal of Crystal Growth, 2003. 253(1-4): p. 297-306.

4. DeLoach, L.D., et al., Vibrational structure in the emission spectra of Yb3+-doped apatite crystals. Journal of Luminescence, 1994. 62(2): p. 85-94.

5. Nelson, A.J., et al., Photoemission and photoabsorption investigation of the electronic structure of ytterbium-doped strontium fluoroapatite. Surface Review and Letters, 2002. 9(1): p. 387-391.

6. Deloach, L.D., et al., Evaluation of Absorption and Emission Properties of Yb-3+ Doped Crystals for Laser Applications. Ieee Journal of Quantum Electronics, 1993. 29(4): p. 1179-1191.

7. Chalmers, B., Principles of Solidification. Wiley, New York, 1964: p. 150.

8. McManus, G.M., R.H. Hopkins, and W.J. Takei, Dislocation Arrangements in Fluorapatite. Journal of Applied Physics, 1969. 40(1): p. 180-\&.

9. $\quad$ Brice, J.C., The cracking of Czochralski-grown crystals. Journal of Crystal Growth, 1977. 42: p. $427-430$.

10. Yu-long Zhang, L.T., Man-made Crystal-growth Technique, Functionality and Application. Chemical Industry Press, China, 2005.

11. Metzger, M. and R. Backofen, Optimal temperature profiles for annealing of GaAs-crystals. Journal of Crystal Growth, 2000. 220(1-2): p. 6-15.

12. Ma, R.H., et al., Integrated process modeling and experimental validation of silicon carbide sublimation growth. Journal of Crystal Growth, 2003. 252(4): p. 523-537.

13. Ma, R.H., et al., Growth kinetics and thermal stress in the sublimation growth of silicon carbide. Crystal Growth \& Design, 2002. 2(3): p. 213-220.

14. Wu, B., et al., Growth kinetics and thermal stress in AlN bulk crystal growth. Journal of Crystal Growth, 2003. 253(1-4): p. 326-339.

15. Tavakkoli, M.H. and H. Wilke, Numerical study of induction heating and heat transfer in a real Czochralski system. Journal of Crystal Growth, 2005. 275(1-2): p. e85-e89.

16. Gevelber, M.A. and G. Stephanopoulos, Dynamics and control of the Czochralski process : I. Modelling and dynamic characterization. Journal of Crystal Growth, 1987. 84(4): p. 647-668.

17. Hurle, D.T.J., et al., The dynamics of czochralski growth. Journal of Crystal Growth, 1990. 100(1-2): p. 11-25.

18. Masi, M., et al., Transient dynamics and control of indium phosphide LEC furnaces. Materials Chemistry and Physics, 2000. 66(2-3): p. 236-245.

19. Irizarry-Rivera, R. and W.D. Seider, Model-predictive control of the Czochralski crystallization process. Part I. Conduction-dominated melt. Journal of Crystal Growth, 1997. 178(4): p. 593-611.

20. Nunes, E.M., et al., A volume radiation heat transfer model for Czochralski crystal growth processes. Journal of Crystal Growth, 2002. 236(4): p. 596-608. 
21. Hossain, M.A. and H.S. Takhar, Radiation effect on mixed convection along a vertical plate with uniform surface temperature. Heat and Mass Transfer, 1996. 31(4): p. 243-248.

22. Ozisik, M.N., Heat Conduction. Wiley, New york, 1980.

23. Song, P., et al., Crystal growth and properties of Yb:FAP laser crystal. Journal of Crystal Growth, 2005. 277(1-4): p. 181-185. 
Table 1. Thermophysical properties of Yb:S-FAP crystal used in the simulation

\begin{tabular}{lc}
\hline Property & Value \\
\hline Density, $\rho\left(\mathrm{g} / \mathrm{cm}^{3}\right)$ & 4.14 \\
Heat capacity, $C_{p}(\mathrm{~J} / \mathrm{gK}) @ 298 \mathrm{~K}$ & 0.5 \\
Thermal diffusivity, $\alpha\left(\mathrm{m}^{2} / \mathrm{s}\right)$ & $9.8 \times 10^{-7}$ \\
Thermal conductivity, $\boldsymbol{k}_{s}(\mathrm{~W} / \mathrm{mK})$ & 2.0 \\
\hline
\end{tabular}

Table 2. Important results for the simulation in Fig. 9

\begin{tabular}{|c|c|c|c|}
\hline Time (hour) & T (K) & Power (\%) & $\Delta \mathrm{T}(\mathrm{K})$ \\
\hline 24 & 1276 & 11.86 & 5.70 \\
75 & 877 & 3.54 & 4.72 \\
150 & 720 & 2.01 & 1.04 \\
\hline
\end{tabular}




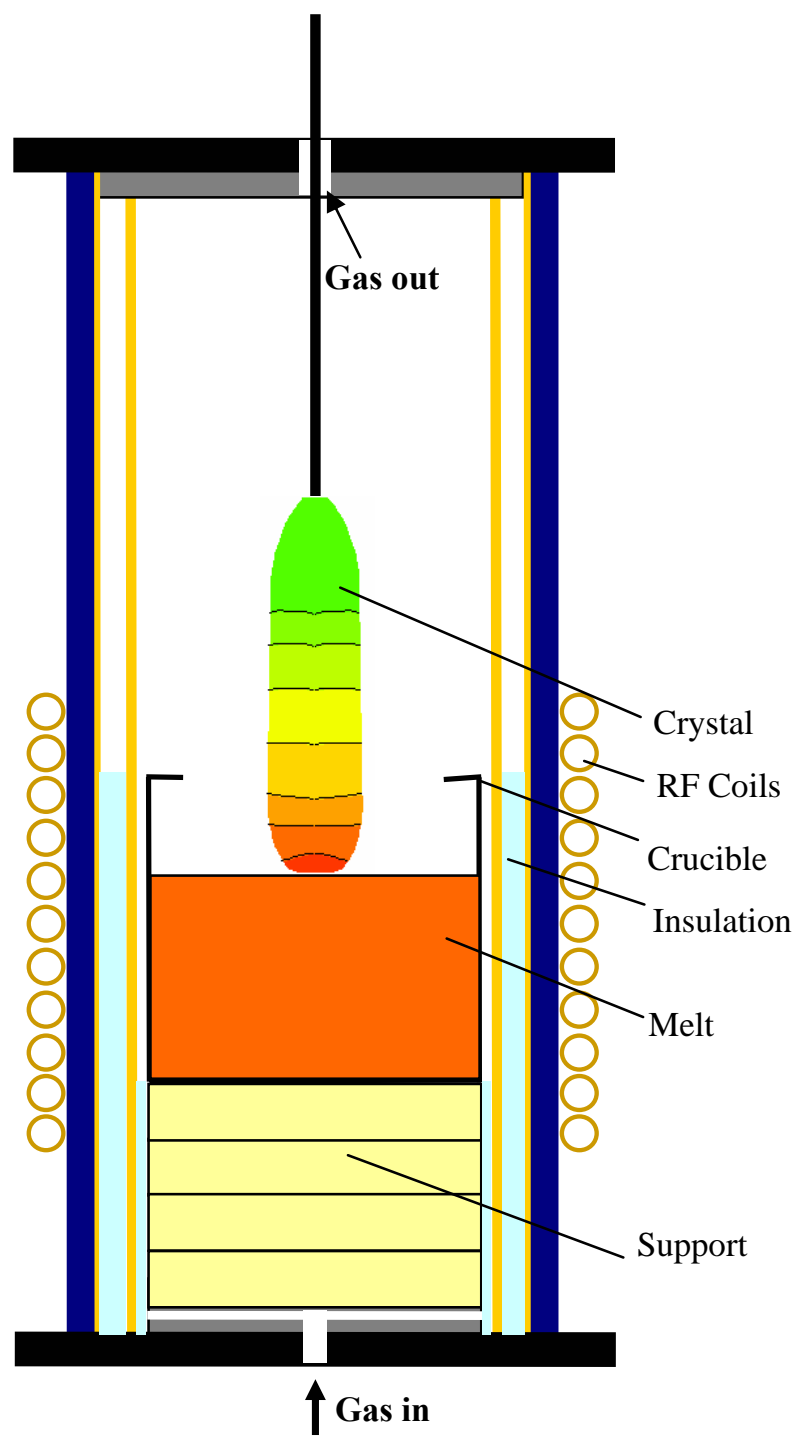

Figure 1. Schematic of the growth furnace 


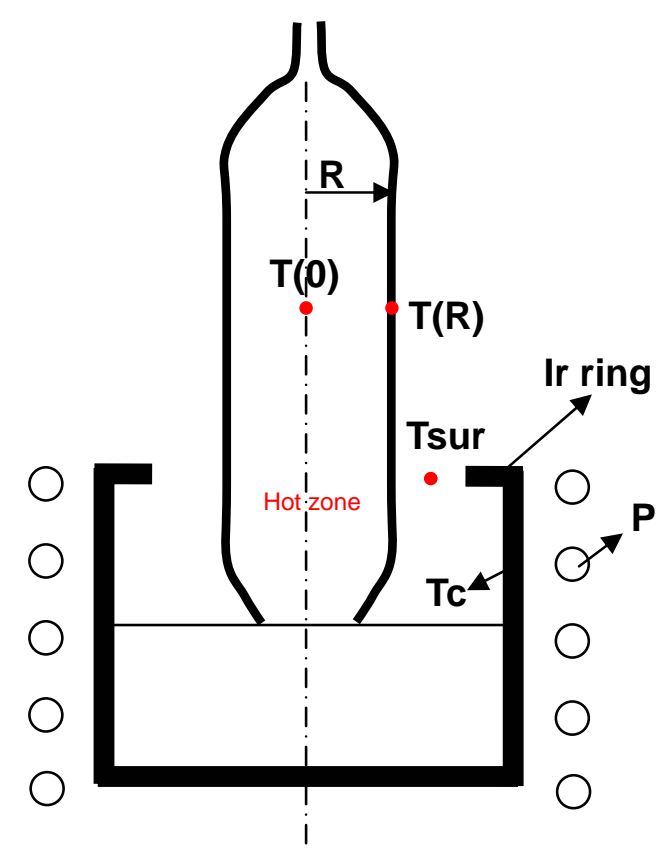

Figure 2. Schematic of the simplified crystal cooling process. Tc: Crucible temperature, Tsur: Crystal surrounding temperature, $T(0)$ : Crystal center temperature, and T(R):

Crystal surface temperature 


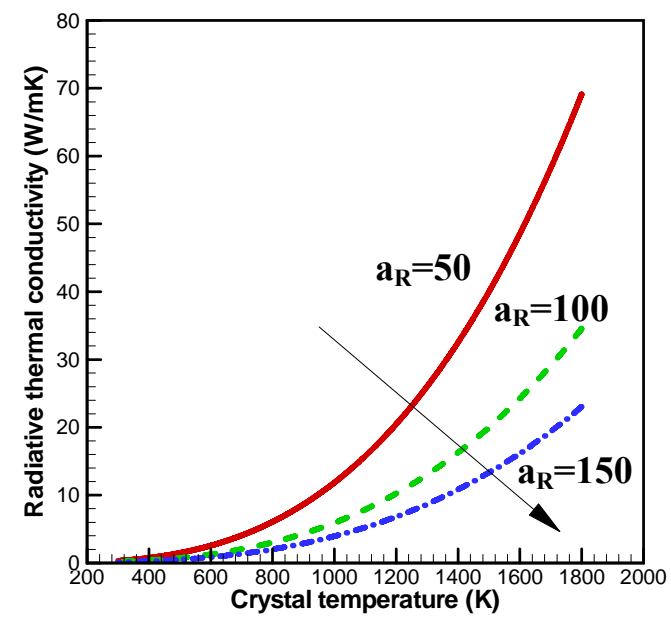

Figure 3. Effect of radiation heat transfer on effective thermal conductivity 


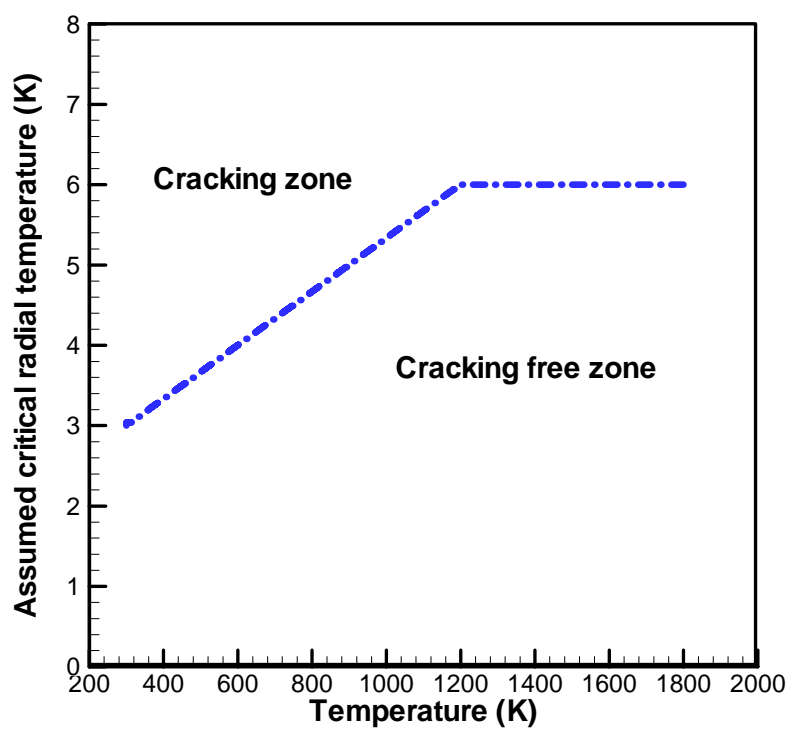

Figure 4 . The critical radial temperature difference used in the simulations.

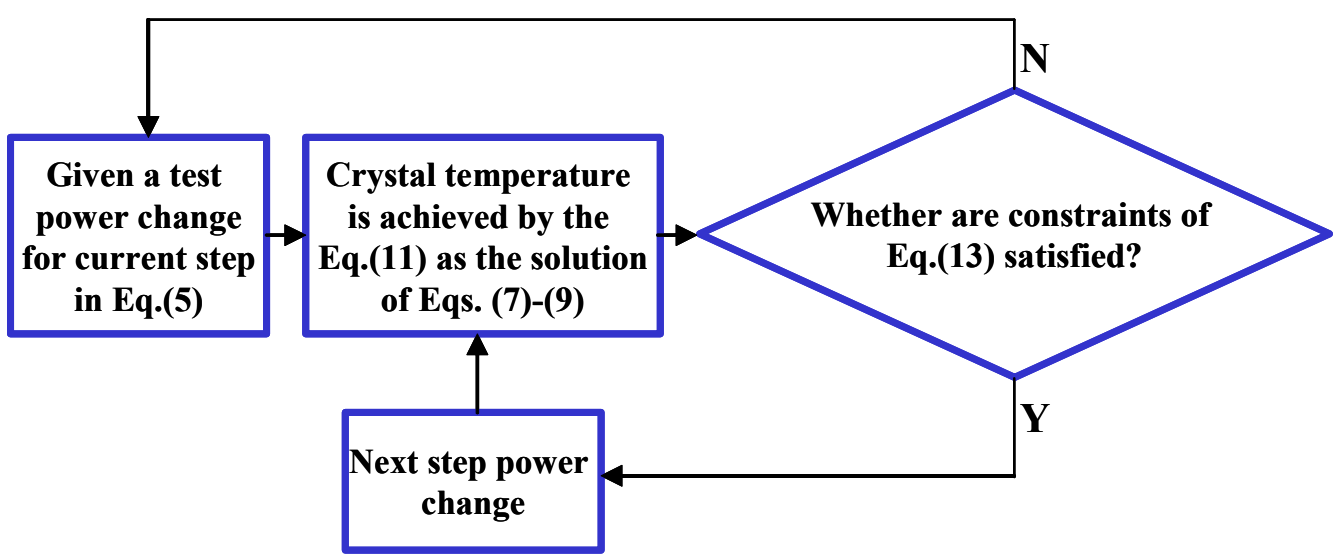

Figure 5. Flowchart of the optimizing procedure 


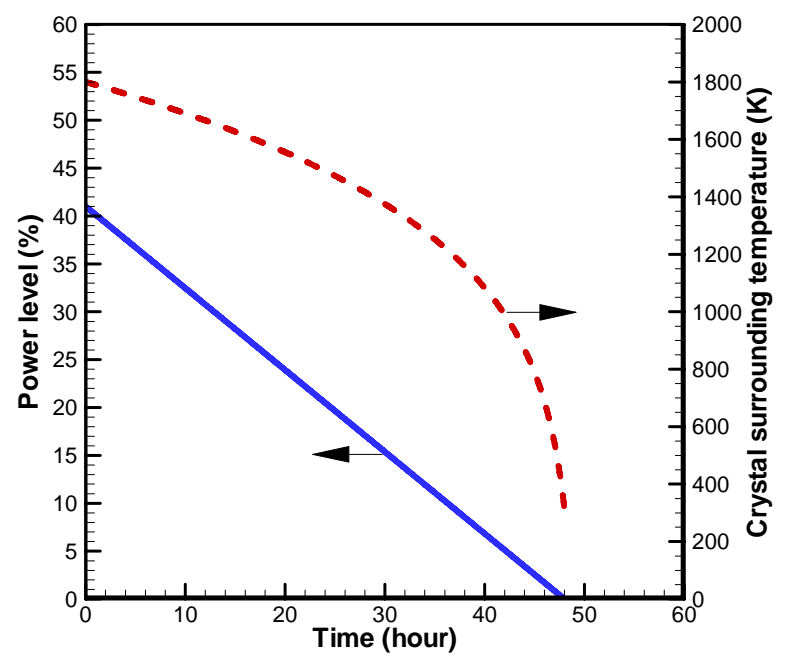

(a)

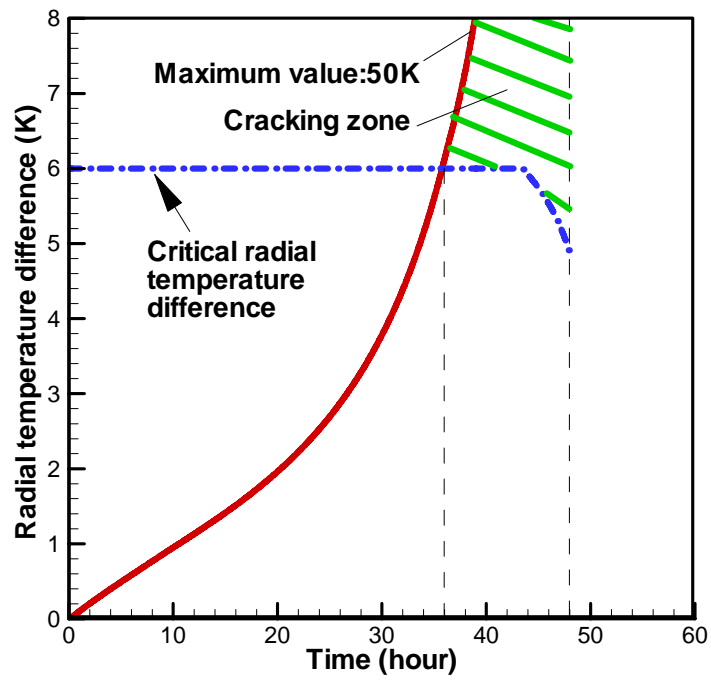

(b)

Figure 6. (a) Linear power ramp-down profile and surrounding gas temperature change profile, and (b) radial temperature difference induced in the crystal. 


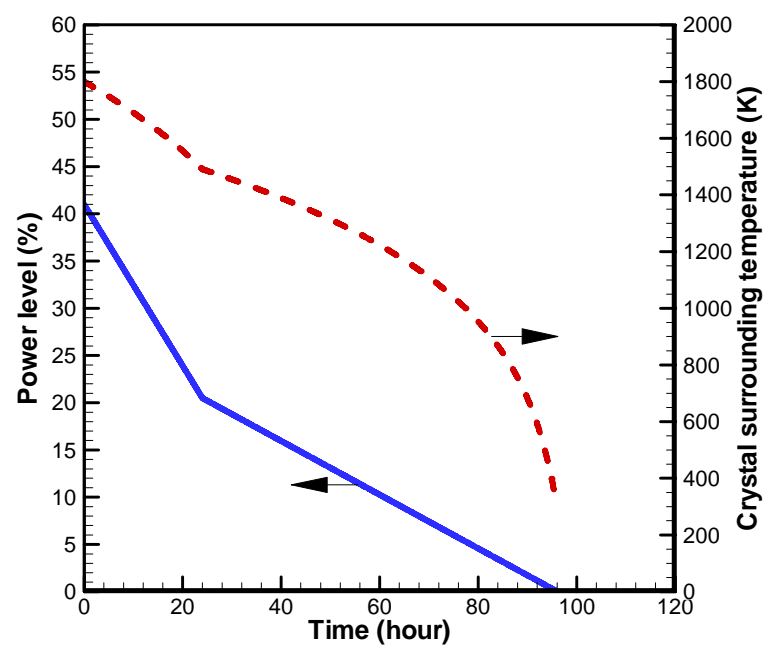

(a)

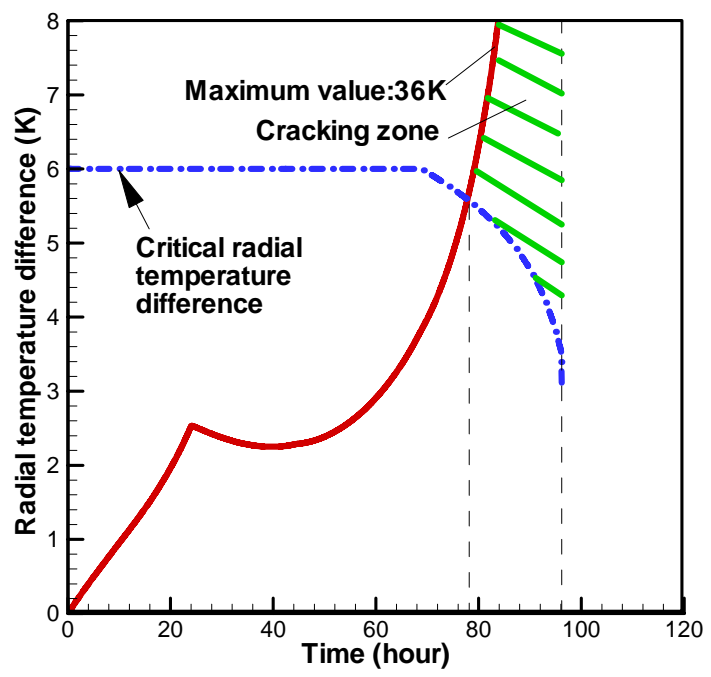

(b)

Figure 7. (a) Bi-linear power ramp-down profile and surrounding gas temperature change profile, and (b) radial temperature difference induced in the crystal 


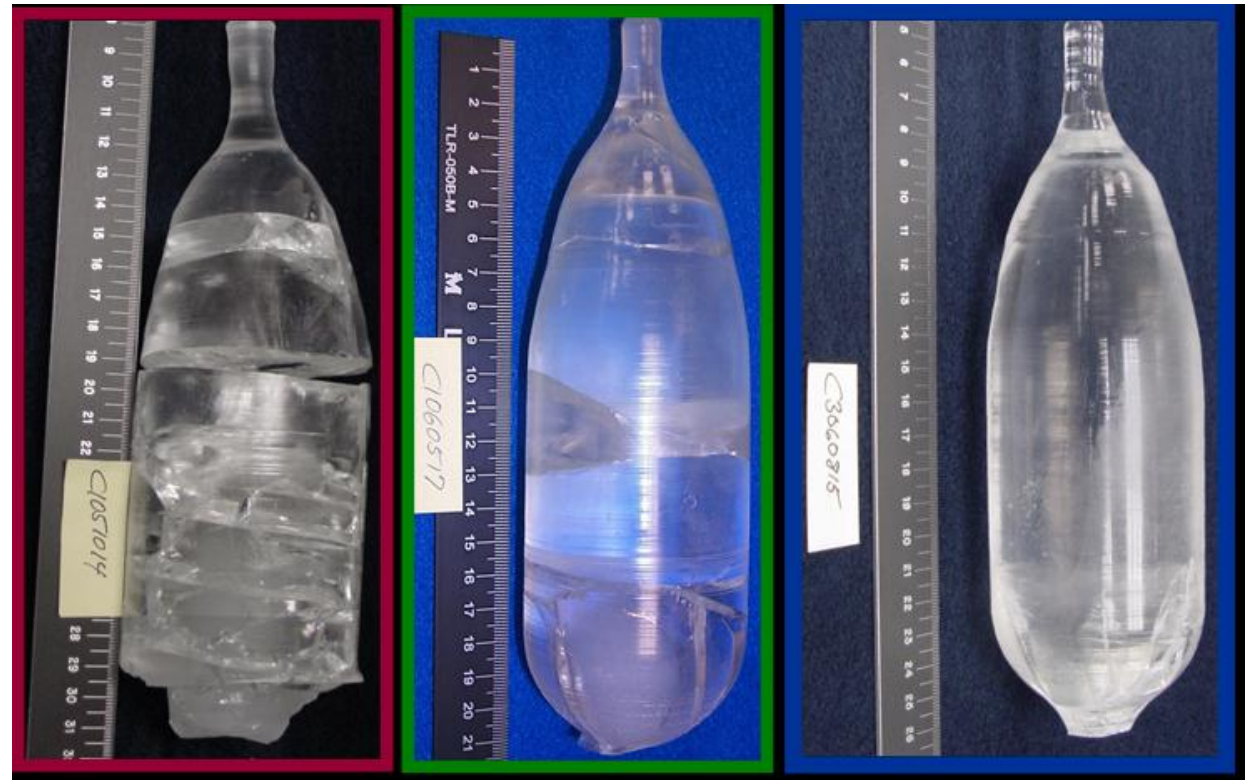

(a)

(b)

(c)

Figure 8. The grown crystals based on the power ramp-down profiles in (a) Fig 6, (b) Fig. 7, and (c) Fig. 10. 


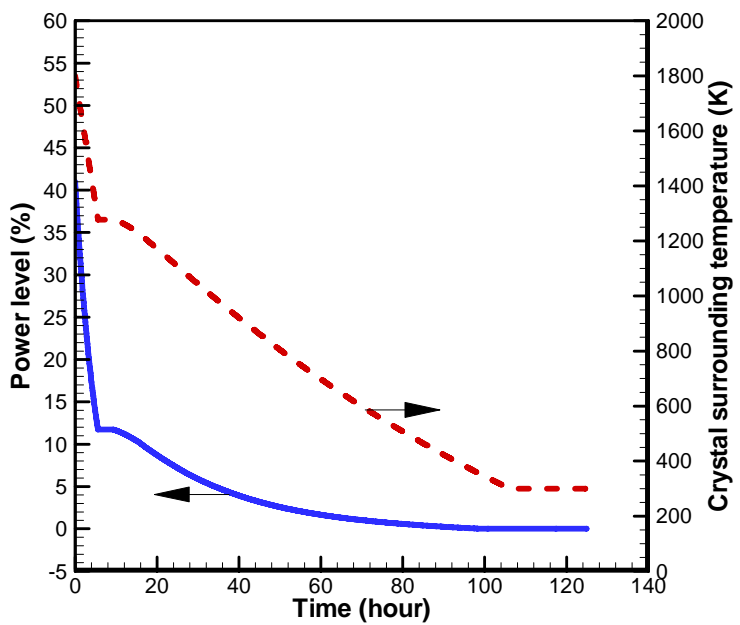

(a)
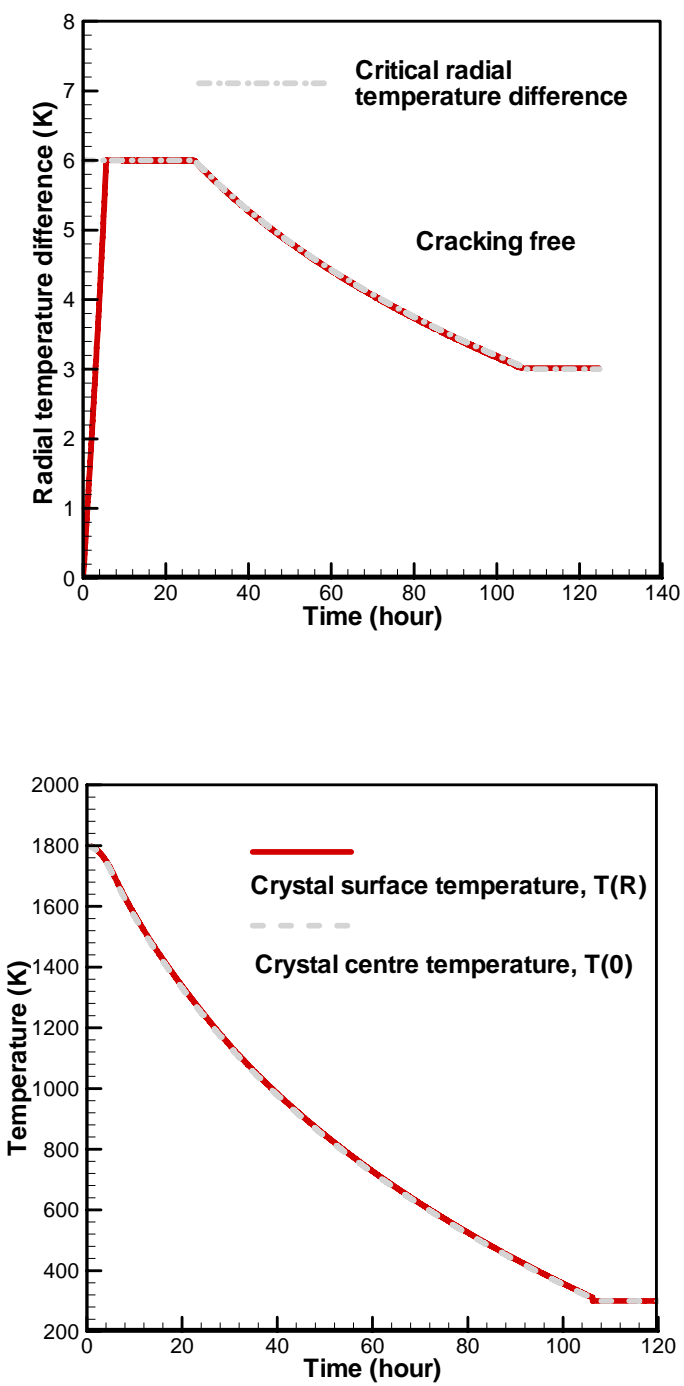

(b) 
Figure 9. (a) Optimized power ramp-down profile and surrounding gas temperature change profile, (b) radial temperature difference induced in the crystal, and (c) crystal surface and center temperature profiles 


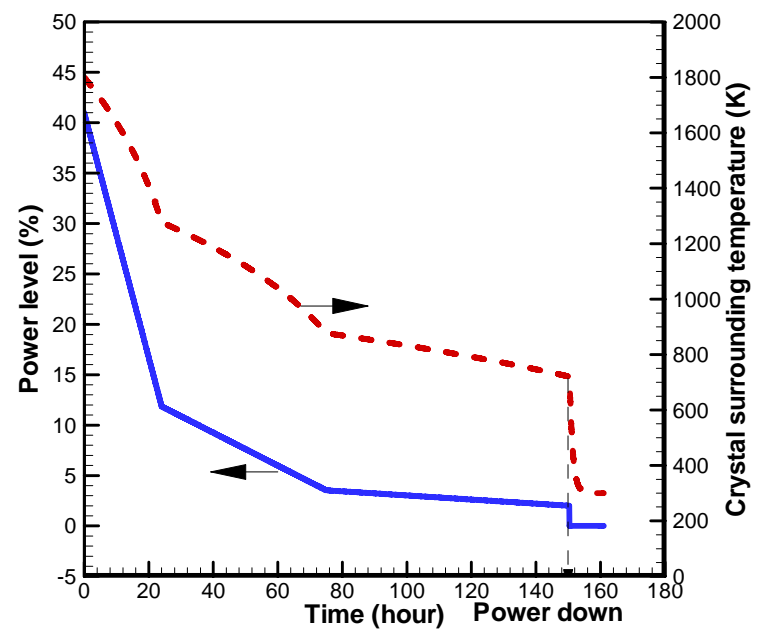

(a)

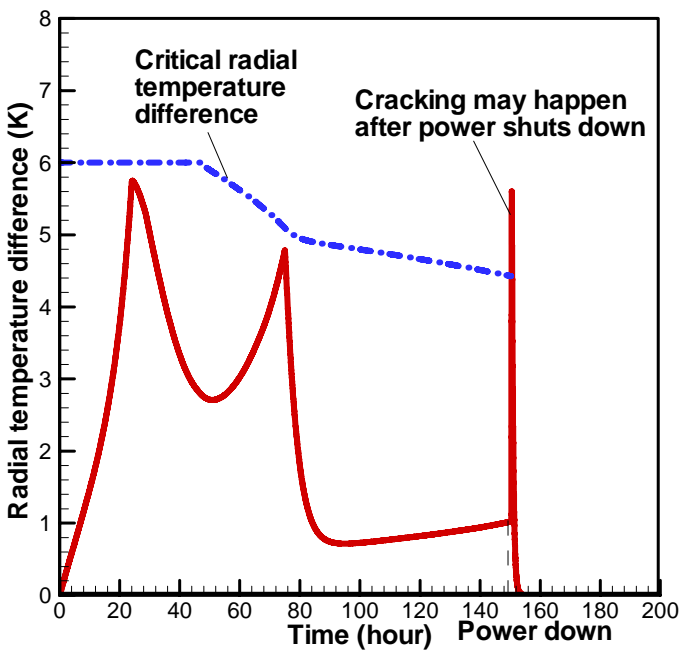

(b)

Figure 10. (a) Tri-linear power ramp-down profile and surrounding gas temperature change profile, and (b) radial temperature difference induced in the crystal 